\title{
Factors associated with adherence to diabetes care recommendations among children and adolescents with type I diabetes: a facility-based study in two urban diabetes clinics in Uganda [Corrigendum]
}

\author{
Kyokunzire C, Matovu N. Diabetes Metab Syndr Obes. \\ 2018;11:93-104.
}

On page 93, Roy William Mayega should have been included as a co-author and was erroneously missed off the authors list.

The author list and affiliations should have been listed as follows:

\section{Catherine Kyokunzire' \\ Nicholas Matovu 2,3 \\ Roy William Mayega ${ }^{4}$}

'Department of Community Health and Behavioural Sciences, School of Public Health, College of Health Sciences, Makerere University, Kampala, Uganda; ${ }^{2}$ Department of Community Health, Division of Noncommunicable Diseases, Ministry of Health - Uganda, Kampala, Uganda; ${ }^{3}$ Global Health Corps Fellowship Program 2017/2018, New York, NY, USA; ${ }^{4}$ Department of Epidemiology and Biostatistics, School of Public Health, College of Health Sciences, Makerere University, Kampala, Uganda

On page 103, Acknowledgements section, "We thank the staff at Mulago Hospital and St Francis Nsambya Hospital diabetes clinics for the support during the study. The participants who took part in the study are also appreciated" should have read "We thank the staff at Mulago Hospital and St Francis Nsambya Hospital diabetes clinics for the support during the study. The participants who took part in the study are also appreciated. We would also like to thank the Makerere University School of Public Health for providing technical support to the team".

On page 103, Author contributions section, "CK helped to conceptualize the design of the project, supervised the data collection, and helped to prepare and revise the final manuscript. NM assisted in conceptualizing the design of the study, carried out the data analysis, interpreted the data, prepared the first draft of the manuscript, and revised the final document. Both authors are responsible for the accuracy and integrity of the presented findings in this study" should read "CK helped to conceptualize the design of the project, supervised the data collection, and helped to prepare and revise the final manuscript. NM assisted in conceptualizing the design of the study, carried out the data analysis, interpreted the data, prepared the first draft of the manuscript, and revised the final document. RWM assisted in the protocol design, modified and reviewed data collection methods, reviewed essential aspects of the study proposal, reviewed comments from the International Review Board (IRB), and helped prepare early drafts of the research report. All authors are responsible for the accuracy and integrity of the presented findings in this study".

The authors wish to apologize for this error. 